\title{
Pensamiento algebraico: Una experiencia de aula, apoyada por TIC, sobre ecuaciones lineales con estudiantes de grado noveno ${ }^{1}$
}

\section{Algebraic Thinking: A classroom experience, supported by ICT, linear equations ninth grade students}

\author{
Pensamento algébrico: Uma experiência em sala de aula, apoiado \\ pelas TIC, equações lineares estudantes nona série
}

Recibido: mayo de 2013

Aceptado: agosto de 2013
Diana Marcela Rincón Hernández ${ }^{2}$

\section{Resumen}

La investigación estuvo orientada a reconocer e impactar positivamente los procesos cognitivos de estudiantes de grado noveno relacionados con el razonamiento algebraico, a partir del diseño e implementación de una secuencia didáctica apoyada por recursos TIC que fueron encontrados y seleccionados en la web mediante criterios analíticos. El estudio correspondió a un diseño de investigación - acción, realizado en cuatro fases (algunas simultáneas): reconocimiento del contexto, diseño, implementación y análisis. Los principales resultados se evidenciaron en los cambios positivos presentados por los estudiantes frente al desarrollo de habilidades en el razonamiento algebraico. Estos se presentaron bajo el análisis de cuatro categorías: recursos TIC, procesos de diseño e implementación, impacto en el aprendizaje y la enseñanza y el contexto social.

Palabras clave: Razonamiento algebraico; aula; recursos didácticos; recursos informáticos; ambiente de aprendizaje apoyado por TIC; matemáticas escolares; álgebra; ecuaciones; ecuaciones lineales; metodología investigación acción.

\section{Abstract}

The investigation was designed to recognize and positively impact the cognitive processes of ninth grade students related to algebraic reasoning, from the design and implementation of a teaching sequence supported by ICT resources were found and selected the Web using analytical criteria. The study corresponded to a research design - action, carried out in four phases (some simultaneously): recognition of the context, design, implementation and analysis. The main results are evident in the positive changes made by students against the development of algebraic reasoning skills. These analyzes were presented under four categories: ICT resources, design and implementation processes, impact on learning and teaching and social context.

1 Artículo de Investigación

2 Universidad de Los Andes. Magister en Educación. Bogotá, Colombia. Contacto: dianamarh@gmail.com 
Keywords: Algebraic Thinking; classroom teaching resources, computing resources, learning environment supported by ICT school math, algebra, equations, linear equations; action research methodology.

\section{Resumo}

A investigação foi concebido para reconhecer e impactar positivamente os processos cognitivos dos alunos do nono relacionadas ao raciocínio algébrico, desde a concepção e implementação de uma seqüência de ensino apoiados por recursos de TIC foram encontrados e selecionados na Web usando critérios de análise. O estudo correspondeu a um projeto de pesquisa - ação, realizada em quatro fases (algumas ao mesmo tempo): o reconhecimento do contexto, design, implementação e análise. Os principais resultados são evidentes nas mudanças positivas feitas por estudantes contra o desenvolvimento de habilidades de raciocínio algébricas. Essas análises foram apresentados em quatro categorias: os recursos de TIC, processos de design e implementação, impacto na aprendizagem e ensino e contexto social.

Palavras-chave: pensamento algébrico, recursos de ensino em sala de aula, os recursos de informática, ambiente de aprendizagem apoiado pelas TIC de matemática da escola, álgebra, equações, equações lineares, metodologia de pesquisa-ação.

\section{Contextualización}

El estudio hizo parte de mi proceso formativo llevado a cabo desde la Maestría en Educación de la Universidad de los Andes. Con esta investigación pretendí diseñar, implementar y evaluar una propuesta didáctica que involucrara los procesos de enseñanza - aprendizaje del razonamiento algebraico con estudiantes del grado noveno, apoyada por recursos TIC.

Con este estudio busqué entrelazar dos ideas: por un lado, las dificultades de mis estudiantes al aprender el álgebra, y por el otro, el uso de la tecnología como apoyo a la educación; a partir de la reflexión, el análisis y la práctica, pretendía plantear posibles soluciones a las dificultades de mi aula, que en lo posible sirvieran como un ejemplo para otras experiencias con un escenario similar.

La importancia que se supone adquiere el álgebra para los estudiantes, viene dada por dos líneas: la primera, como un elemento que es necesario aprender a usar para apoyar a otros conceptos matemáticos; la segunda, como un lenguaje novedoso que aporta a la comunicación desde las matemáticas (Esquinas, 2009). Desde este sentido, abordar la enseñanza y el aprendizaje del álgebra toma un papel de gran relevancia, en cuanto a las bondades que su desarrollo le va a brindar a cada estudiante.

La población con la que se desarrolló el estudio fue un grupo de estudiantes del grado noveno, cuyas edades oscilaron entre los 12 y 18 años. Con el fin de analizar en detalle el proceso y el progreso de una muestra de estudiantes, se escogió un grupo de 4 hombres y 2 mujeres. Fueron elegidos pretendiendo tener dos estudiantes de cada nivel de desempeño en matemáticas, alto, medio y bajo, tras haber aplicado una prueba inicial.

\section{Referentes teórico-prácticos básicos}

En la planeación, implementación y evaluación de cualquier actividad de enseñanza - aprendizaje se debe reflexionar sobre diversas preguntas que facilitan este tipo de procesos: ¿qué?, ¿cómo?, ¿cuándo?, ¿con qué?, ¿para quién?, ¿para qué 
enseñar? (Marín, Flores \& Lupiañez, 2006); éstas son las principales preguntas que busqué responder a través de los apartados sobre el razonamiento algebraico para grado noveno, con el fin de apoyar la secuencia didáctica que se planteo. La revisión conceptual la realicé desde cuatro enfoques: [1] enseñanza del álgebra: niveles cognitivos, dificultades o errores en el aprendizaje del álgebra, retroalimentación; [2] recursos didácticos para álgebra, [3] recursos tecnológicos para el apoyo de procesos de aprendizaje: ambientes de aprendizaje apoyados con tecnología y objetos virtuales de aprendizaje y finalmente, [4] educación matemática en situaciones de conflicto social y político.

\section{Descripción general de la experiencia de aula}

La intervención se realizó en 12 sesiones. El propósito de uso del recurso TIC se enfocaba en ayudar a los estudiantes a representar gráficamente los conceptos matemáticos, con el fin de realizar manipulaciones o modelización de algunas situaciones y objetos. Así mismo se buscaba que los estudiantes plantearan conjeturas, hicieran pruebas y finalmente construyeran generalizaciones. La intervención se diseñó con el tema "ecuaciones simples". La siguiente gráfica describe la secuencia de actividades de aprendizaje:

Gráfica 1: Secuencia de aprendizaje.

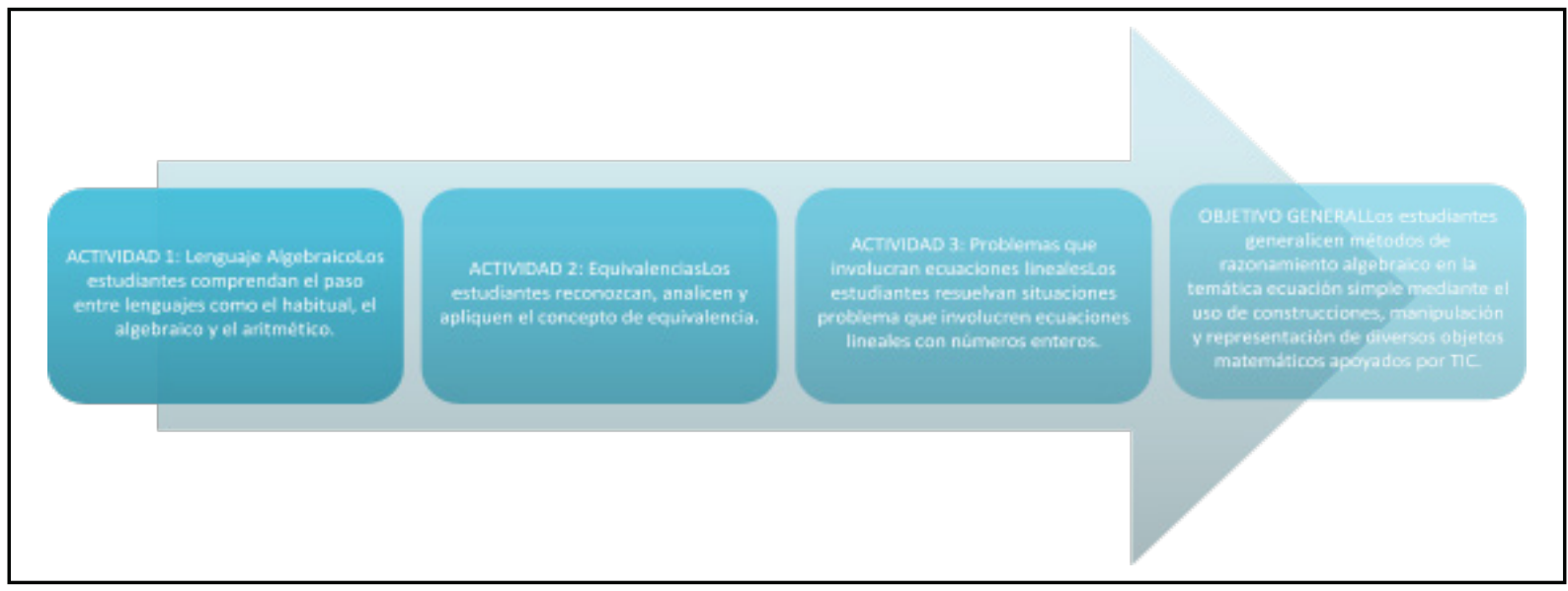

Fuente: Elaboración propia.

\section{Logros y dificultades}

Logros. Los logros obtenidos en esta intervención se encuentran en tres aspectos: Usos de los recursos tecnológicos como apoyo a los procesos de enseñanza - aprendizaje, impacto en el aprendizaje de los estudiantes, impacto en mi práctica educativa, entre algunos se encuentran:

- El uso de recursos tecnológicos aportó diferentes bondades al proceso de aprendizaje: (i) por medio de la manipulación de simulaciones los estudiantes exploraron conjeturas para llegar a conclusiones sobre los temas de equivalencias y solución de problemas que involucran ecuaciones lineales; (ii) las herramientas tecnológicas brindaron un elemento concreto para los estudiantes sobre el cual discutir con sus compañeros y profesora. Además, los recursos tecnológicos brindaron (iii) un proceso de retroalimentación diferente, en cuanto a la propia retroalimentación dada por cada recurso y la dada en el blog de cada estudiante.

- El análisis de progreso de cada estudiante entre la prueba inicial y la prueba final - evidencia que el $83 \%$ de los estudiantes (5 de 6 ), cambiaron positiva y significativamente su desempeño en la prueba. Los componentes uso de la letra, generalización y solución de problemas que involucran ecuaciones 
lineales, fueron los elementos que presentaron mayor cambio en el desempeño de los estudiantes.

- Un elemento que no había sido planeado, pero que con el trascurso del estudio se presentó como fundamental, fue la comunicación o expresión verbal de los estudiantes. Esta competencia se tornó un elemento decisivo para mejorar la comprensión de los estudiantes sobre los conceptos matemáticos aquí abordados. Lo comunicativo se pudo observar desde dos perspectivas: el uso del lenguaje para explicar su comprensión del tema y su uso para discutir argumentos con sus compañeros o profesora.

- En mi práctica educativa este estudio me brindó mayores herramientas para tomar decisiones argumentadas y enriquecer mi práctica en el aula de clase. Actualmente me cuestiono en mayor profundidad aspectos como: ¿cuál o cuáles son las habilidades o competencias que quiero desarrollar con mis estudiantes en $\mathrm{x}$ grado? y ¿cuáles son los conocimientos esenciales del grado en el que se encuentran? Tomo estos elementos como punto de partida para mi planeación en cuanto a objetivos, actividades, uso de recursos, evaluación, entre otros.

- Dificultades. Las dificultades presentadas en la intervención se recogen en dos aspectos: Técnicos y cognitivos:

- Los componentes de simplificación de expresiones algebraicas y multiplicación de expresiones algebraicas no tuvieron un cambio positivo tan representativo como otros desempeños. Considero que esta situación se presenta por elementos como: [1] Poco tiempo de interacción entre los estudiantes y la intervención, aunque la implementación duró aproximadamente mes y medio ${ }^{3}$, los procesos de aprendizaje suelen requerir de más tiempo y variedad de actividades para que sean reestructurados. [2] Las actividades no estaban enfocadas directa y explícitamente para mejorar este desempeño. Probablemente para este tipo de situaciones es necesario plantear actividades más particulares para que los estudiantes comprendan el manejo del algoritmo que se presenta. [3] Los conocimientos previos no desarrollados influyen en la construcción de los nuevos conocimientos (Coll, Martín, Mauri, Miras, Onrubia, Solé, \& Zabala, 1994).

- El no poder usar el blog generó cambios en lo planeado, puesto que los estudiantes no lograron, por problemas técnicos, hacer uso del mismo dentro del espacio brindado por el colegio; por lo tanto, fue necesario cambiar la forma de presentar las reflexiones de los estudiantes (en un trabajo enviado por mail o en un trabajo presentado en el aula) lo que generó que se perdiera la posibilidad de compartir estas reflexiones entre compañeros.

- Conflictos sociales que afectan negativamente en las instituciones educativas frecuentemente generan nuevos elementos de reflexión y necesidad de hacer cambios en las planeaciones didácticas de los docentes. Un ejemplo: La toma por los estudiantes de la Institución Educativa José Francisco Socarrás durante la intervención de este estudio.

\section{Reflexión final}

Una invitación de Fierro, Fortoul y Rosas (1999) es a transformar las prácticas pedagógicas a través de la investigación - acción, como elemento para reflexionar sobre la práctica docente de cada uno. Entre las dimensiones que ellas proponen analizar se encuentra la dimensión didáctica, la cual fue impactada en una mayor medida en este estudio, al respecto he logrado una reconstrucción de mi desempeño docente, un elemento que no me permite en la actualidad tomar decisiones de aula sin

3 En tiempo real un mes, teniendo en cuenta el tiempo perdido por la toma del colegio. 
pensar en cada uno de los aspectos en los que posiblemente intervendría.

Un elemento con el cual concluí este estudio fue la reflexión vista como el elemento más importante en la investigación - acción (Latorre, 2007), considero fue uno de los aspectos que más aportó a mi estudio, a mi aprendizaje y a mis decisiones y al mismo tiempo fue el más difícil de realizar. La reflexión suele atravesar la investigación como una espiral reflexiva dentro del estudio y la intervención, también sentí que ocurría este mismo evento en mi proceso como investigadora. Usando la investigación - acción descubrí elementos valiosos para tener en cuenta en los procesos investigativos pero también descubrí mis debilidades, en las que debo enfocarme para mejorar mis prácticas y especialmente mi reflexión sobre mis desempeños en el aula.

\section{Referencias}

Esquinas, A. (2009). Dificultades de aprendizaje del lenguaje algebraico: del símbolo a la formalización algebraica: aplicación a la práctica docente. Tesis Doctoral Universidad Complutense de Madrid.

Coll, C., Martín, E., Mauri, T., Miras, M., Onrubia, J., Solé, I. \& Zabala, A. (1993). El constructivismo en el aula. Barcelona: Editorial Graó.

Fierro, C., Fortoul, B. \& Rosas, L. (1999). Transformando la práctica docente: Una propuesta basada en la investigación - acción. México D.F.: Paidós. Maestros y Enseñanza.

Latorre, A. (2007). La investigación-acción: Conocer y cambiar la práctica educativa. Barcelona: Grao.

Marín, A., Flores, P. \& Lupiañez, J. (2006). Materiales $y$ recursos en el aula de Matemáticas de Secundaria y Bachillerato. Cursos de formación a distancia Thales-CICA 2006-07. 